\title{
Review Article \\ Characteristics of HLA-E Restricted T-Cell Responses and Their Role in Infectious Diseases
}

\author{
Simone A. Joosten, ${ }^{1}$ Lucy C. Sullivan, ${ }^{2}$ and Tom H. M. Ottenhoff ${ }^{1}$ \\ ${ }^{1}$ Department of Infectious Diseases, Leiden University Medical Center, 2333 ZA Leiden, Netherlands \\ ${ }^{2}$ Department of Microbiology and Immunology, The University of Melbourne, Peter Doherty Institute for Infection and Immunity, \\ Melbourne, VIC 3010, Australia \\ Correspondence should be addressed to Simone A. Joosten; sajoosten@lumc.nl
}

Received 18 April 2016; Revised 28 June 2016; Accepted 10 July 2016

Academic Editor: Nathalie Rouas-Freiss

Copyright (C) 2016 Simone A. Joosten et al. This is an open access article distributed under the Creative Commons Attribution License, which permits unrestricted use, distribution, and reproduction in any medium, provided the original work is properly cited.

Human HLA-E can, in addition to self-antigens, also present pathogen-derived sequences, which elicit specific T-cell responses. T-cells recognize their antigen presented by HLA-E highly specifically and have unique functional and phenotypical properties. Pathogen specific HLA-E restricted $\mathrm{CD}^{+}$T-cells are an interesting new player in the field of immunology. Future work should address their exact roles and relative contributions in the immune response against infectious diseases.

\section{Introduction}

T-cell activation requires specific recognition of antigen presented as small fragments (peptides) bound to major histocompatibility complex (MHC) molecules. The recognition of a particular peptide-MHC occurs through a highly specific T-cell receptor (TCR), which is selected in the thymus. Following TCR triggering, costimulation and the presence of polarizing cytokines together determine the T-cell activation pattern and guide ultimate T-cell differentiation. Classically, $\mathrm{CD} 4^{+} \mathrm{T}$-cells recognize antigens scavenged extracellularly by the antigen presenting cell (APC) that are presented in MHC class II, whereas $\mathrm{CD} 8^{+} \mathrm{T}$-cells recognize endogenous antigens presented by MHC class I (MHC-I) [1, 2]. In spite of this widely held view, already decades ago it was shown that also antigens derived from intracellular pathogens such as viruses or intracellular bacteria can be presented in MHC-I [3]. More recently, cross-presentation by dendritic cells and autophagy have been elucidated as important mechanisms in this context $[2,4]$.

Transplantation of hematopoietic cells as well as solid organs and detailed studies of viral infections provided the initial key information leading to the concept of genetic MHC restriction by autologous MHC molecules. This is currently often referred to as "conventional" or "donorrestricted" immunity [5]. However, numerous T-cell subsets have been identified that do not fulfil these criteria, including MHC class Ib restricted T-cells, CD1 restricted T-cells, MR1 restricted mucosal associated invariant T-cells (MAIT), NKT-cells, and $\gamma \delta$ T-cells, subsets that are collectively called "unconventional" or "donor-unrestricted T-cells" (DURT) [5]. Unconventional T-cells behave differently in terms of memory, kinetics, and ligands recognized compared to conventional T-cells as recently summarized [5].

An intriguing group of DURT family cells are the Tcells that are restricted by MHC class Ib molecules. These cells may share several critical properties with conventional T-cells but most importantly recognize antigens typically in the context of nonpolymorphic MHC-I molecules. The human MHC class Ib family, also called nonclassical HLA class I, is comprised of HLA-E, HLA-F, and HLA-G. The major difference with classical class Ia molecules is their very low level of allelic variation. Whereas HLA class Ia families are composed of several hundred family members for HLA-A, HLA-B, and HLA-C alleles, HLA-E, HLA-F, and HLA-G comprise only 3, 4, and 10 family members, respectively, and not all of these are actually expressed as functional proteins [6]. Immune cells express relatively high 


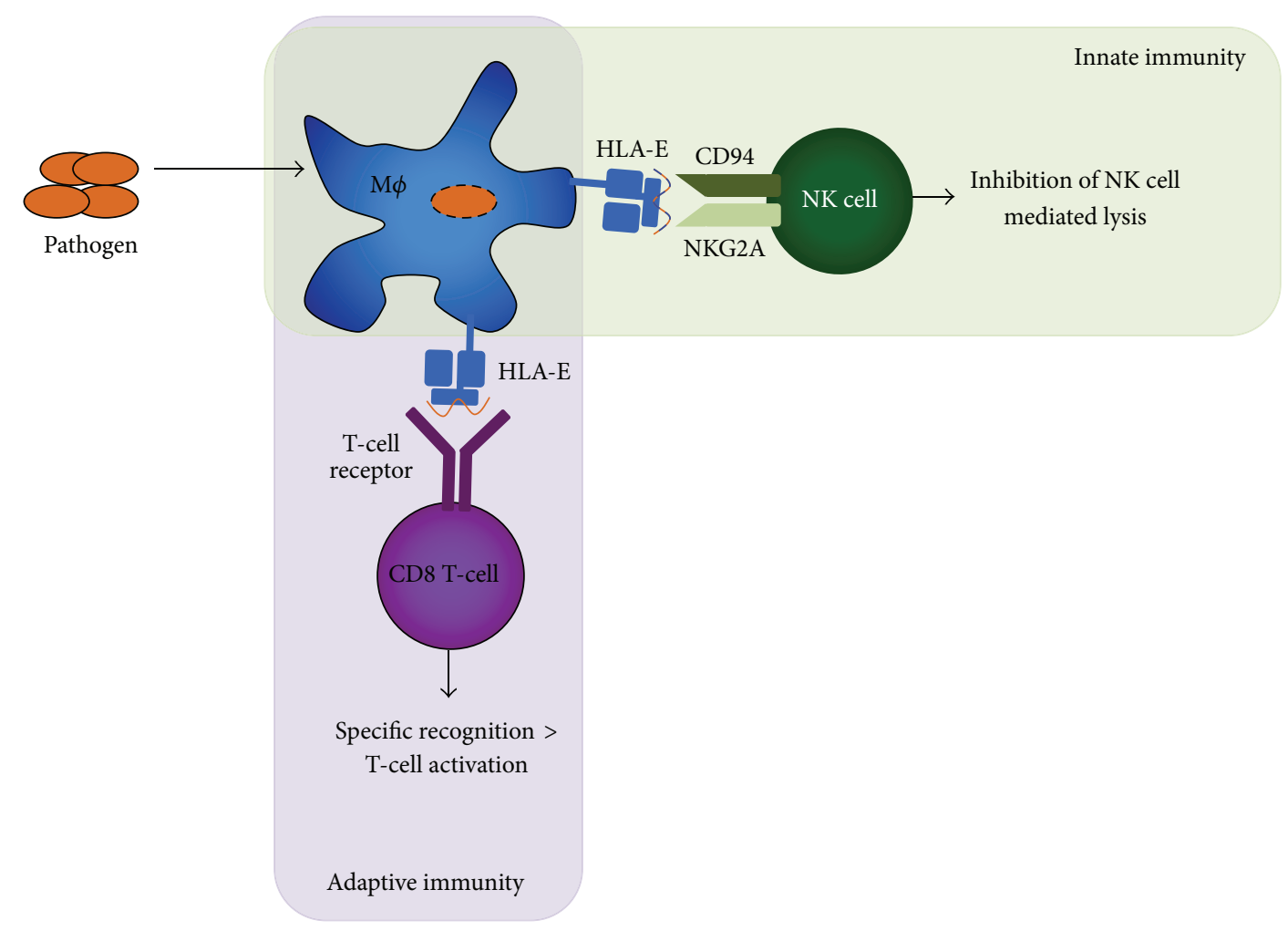

FIGURE 1: HLA-E serves a dual role in the immune system. HLA-E presents antigens, including pathogen-derived antigens on the cell surface of most cells. NK cells, as part of the innate immune system, will sense the presence of HLA-E presenting self or pathogen-derived peptides and thereby receive inhibitory signals from the CD94/NKG2A complex such that NK mediated lysis will be inhibited. In addition, CD8 ${ }^{+}$ T-cells may specifically recognize foreign peptide presented by HLA-E and become activated through their T-cell receptor, resulting in T-cell activation, expansion, and memory formation in the adaptive immune system.

levels of HLA-E protein, but also tissue cells can express the HLA-E protein (http://www.proteinatlas.org/). Although HLA-E was originally described to be broadly expressed by almost all cells that also express HLA class Ia molecules [7], other studies suggest HLA-E expression is restricted to lymphoid and endothelial cells [8]. Furthermore, pathogens can affect HLA-E cell surface expression; for example, human cytomegalovirus (CMV) can upregulate its expression [9]. HLA-E functions as ligand for CD94-NKG2 receptors and has a peptide-binding groove that is ideally suited for binding peptides derived from the leader sequences of other MHCI molecules [10]. In this regard, the loss of leader-peptide loaded HLA-E expression is a marker for cells having lost expression of HLA class Ia molecules, which targets these cells for recognition and lysis by Natural Killer (NK) cells [10]. In contrast to HLA-E, HLA-F expression appears to be more restricted and is detected mostly in liver and bladder [10]. However, its expression is largely intracellular and in association with other MHC-I molecules, which has led to speculations that HLA-F might be involved in the intracellular stabilization of HLA class Ia molecules [10]. The third human MHC class Ib family member, HLA-G, has an even more narrow tissue distribution; its expression appears limited to trophoblasts in the placenta, and it has been associated with fetal-maternal tolerance [10]. HLA-G may function during pregnancy to inhibit NK mediated lysis as trophoblasts lack HLA-A and HLA-B expression [11].

Thus, given the intracellular expression of HLA-F and the placental restriction of HLA-G, limited information is available on T-cells interacting with these molecules, and their relevance to general immunity remains unclear. For this reason, the focus of this review will be on HLA-E restricted T-cells.

\section{HLA-E}

The role of HLA-E in the innate immune response is to present signal sequence-derived peptides of other HLA class I molecules to inhibit NK mediated lysis of cells via recognition by CD94/NKG2A [13]. However, HLA-E can also bind and present other peptide sequences, which can be self or pathogen derived and can be recognized by adaptive T-cells. HLA-E is thus considered to play a role in both innate and adaptive immunity, via interacting with both NK cells as well as presenting peptides to antigen specific $\mathrm{CD}^{+}{ }^{+} \mathrm{T}$-cells (Figure 1).

Eleven alleles have been reported for HLA-E, only 3 of which can be translated into proteins, 2 of them being highly dominant, the HLA-E ${ }^{\mathrm{R}}\left(\mathrm{E}^{*} 01: 01\right)$ and the HLA-E ${ }^{\mathrm{G}}\left(\mathrm{E}^{*} 01: 03\right)$ variants, which differ only in a single amino acid at position 
107, being arginine ( $\left.E^{*} 01: 01\right)$ or glycine ( $\left.E^{*} 01: 03\right)$. Position 107 is located on the loop between the $\beta$-strands outside of the $\alpha 2$ domain of the heavy chain, just outside the peptide-binding groove. The frequency of the HLA- $\mathrm{E}^{\mathrm{R}}$ and HLA- $\mathrm{E}^{\mathrm{G}}$ in the population is about equal, suggesting balanced selection in diverse populations $[14,15]$. Whether HLA- $\mathrm{E}^{\mathrm{R}}$ and HLA$\mathrm{E}^{\mathrm{G}}$ display functional differences has not been studied in detail $[14,16]$, but it has been demonstrated that HLA-E ${ }^{\mathrm{G}}$ homozygous cells express higher levels of HLA-E and had higher peptide-binding affinity [16]. More recently, peptide elution studies revealed a different peptide-binding repertoire eluted from HLA- $\mathrm{E}^{\mathrm{R}}$ versus HLA- $\mathrm{E}^{\mathrm{G}}$ molecules, indicating that the F-pocket of HLA-E ${ }^{G}$ bound a smaller variety of peptides and had a stronger preference for a lysine at the $\mathrm{p} \Omega$ position $[17,18]$.

2.1. HLA-E Peptide Binding. The structural basis of HLA-E's ability to bind signal sequence-derived peptides from HLA class Ia (HLA-I) molecules has been studied previously. Unlike HLA class Ia that typically contain 2 or 3 anchor residues, HLA-E contains 5 anchor residues in the peptidebinding groove that highly constrains the sequence of the bound peptide $[19,20]$. However, HLA-E was additionally shown to bind peptides derived from viruses such as influenza M1 protein and EBV BZLF1 [21]. Moreover, a sequence from cytomegalovirus (CMV) glycoprotein UL40, which is identical to the HLA-C ${ }^{*} 03$ leader sequence, can bind to HLA-E and is capable of preventing NK mediated lysis $[9,22]$. The same was found for a HCV derived sequence, despite its sequence difference from signal peptides [23]. Peptide identification and characterization using random peptide approaches also revealed that a leucine on $\mathrm{P9}$ is a critical anchor residue for HLA-E binding but did not identify methionine as critical P2 anchor for HLA-E binding and folding [24]. Identification of the motif within the HLA class Ia leader sequences critical for interaction with the CD94/NKG2 complex suggested anchor residues at positions 2, 6, 7, and 9, while solvent exposed residues at P5 and P8 were likely important for binding to CD94/NKG2 [25]. Thus, while P2 and P9 are anchor residues for binding to HLA-E and $\mathrm{P} 5$ and $\mathrm{P} 8$ are involved in the interaction with CD94/NKG2 [26], P8 is also the critical residue distinguishing self (signal sequence) from nonself (CMV UL40) and allowing for TCR recognition of CMV UL40 [12].

Recent peptide elution studies provided important insights in the types of peptides that are naturally presented by HLA-E and identified a larger array of peptides eluted from HLA-E than originally discovered [17, 18, 27]. Eluted peptides were generally short (8-9-10 mers), but occasionally also longer peptides were eluted including 11-17 mers $[17,18,27]$. This is in line with earlier studies, where also peptides greater than 8 amino acids could bind HLA-E [21]. Furthermore, eluted peptides were different from signal sequences, possessing hydrophobic amino acids on P2 and P9, consistent with a binding motif that was very similar to that of HLA-A2 [27]. Recent studies in rhesus macaques immunized with SIV-gag (Simian Immunodeficiency Virus), in specific CMV vectors, revealed a series of peptides that were recognized by $\mathrm{CD} 8^{+} \mathrm{T}$-cells but only a minority contained the canonical MHC class I antigen E (MHC-E) binding motif [28]. Structural analyses revealed that the peptide-binding cleft of HLA-E is rigid but relatively open compared to that of HLA class Ia family members. Peptides that lack the canonical residues can adopt a backbone structure that is similar to canonical peptides, allowing them to bind the HLA-E molecule [28]. These unique binding properties of HLA-E may explain the observed epitope diversity and breadth in SIV-gag in the rhesus macaque vaccination studies [28]. Furthermore, the authors suggested that the open structure of HLA-E may allow peptide exchange [28]; this may be in particular relevant for Mycobacterium tuberculosis (Mtb) as HLA-E expression is enriched in the Mtb phagosome [29]. Intriguingly, we have identified a large series of Mtb epitopes presented in HLA-E and recognized by mycobacteria exposed human donors, many of which lack the canonical residues [30].

Together, these data indicate that HLA-E binds signal sequence-derived peptides not only from MHC class Ia molecules but also from other self and even pathogen-derived sequences. Although many peptides contain canonical amino acids for binding HLA-E, clear examples exist for peptides which lack canonical residues and can still bind HLAE. Presentation of nonself sequences, being absent during thymic selection, may elicit adaptive immune responses by $\mathrm{CD}^{+} \mathrm{T}$-cells.

2.2. HLA-E Restricted T-Cells. Specific recognition of pathogen-derived sequences presented by the unconventional presentation molecule HLA-E by the $\mathrm{CD} 8^{+}$TCR could lead to specific activation of adaptive immune responses, independent of classically HLA restricted $\mathrm{CD}^{+}$and $\mathrm{CD} 8^{+} \mathrm{T}$ cells. In several infectious disease models, evidence for such responses has been reported recently.

2.2.1. Viral Antigens. As viruses require the human host to survive and therefore reside within host cells, their proteins are presented by HLA class I molecules, including HLA class Ib. Peptides from Epstein Barr Virus (EBV) [34-36], Cytomegalovirus (CMV) [12, 37-41], and Hepatitis C Virus (HCV) [44] can be presented by HLA-E and are recognized by virus specific T-cells (Table 1). The peptide epitopes studied from CMV UL40 are highly similar to the HLA class Ia signal sequences, whereas the sequences from EBV BZLF1 and HCV appear more different. The functional and phenotypical description of these T-cells is rather limited, but they all express $\mathrm{CD}^{+}$as expected for HLA class I restricted cells. In many studies, HLA-E restricted T-cells have been identified and enumerated using HLA-E tetramers whereas functional analyses were limited to the demonstration of target cell lysis. Likewise, phenotypical characterizations were very limited in scope but when performed showed a cytolytic Tcell phenotype (perforin, granzyme A/B) and IFN $\gamma$ production in some studies [38, 41].

(1) HLA-E and CMV. Initial studies on possible recognition of HLA-E peptide complexes by T-cells in a TCR dependent manner were performed using signal sequences from HLA 


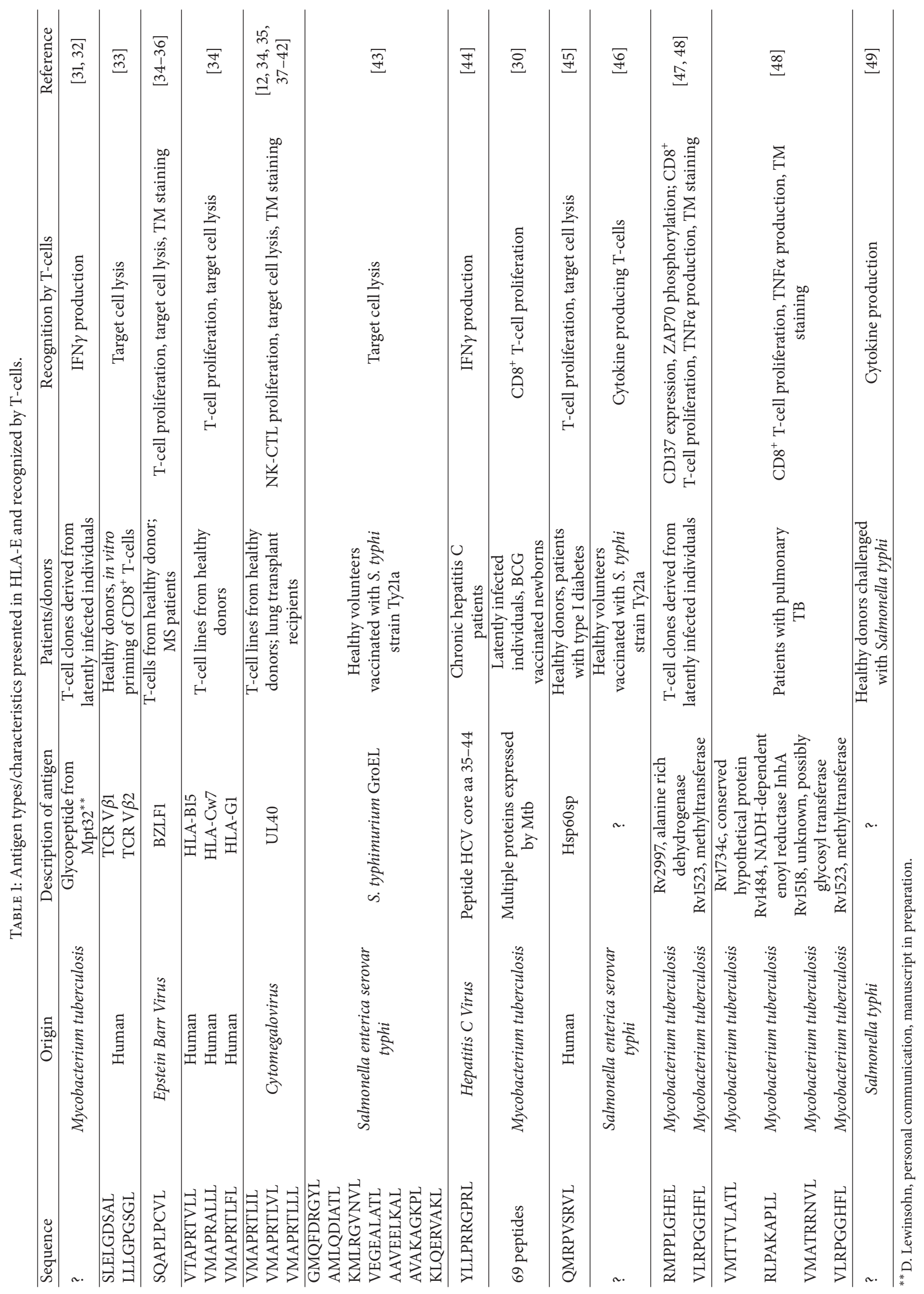




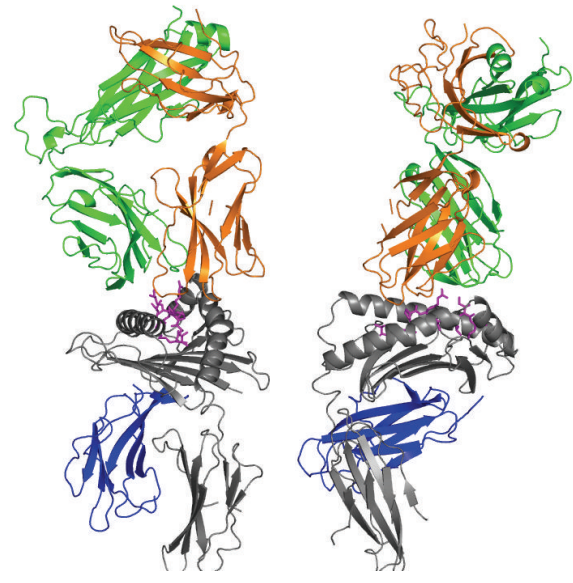

(a)

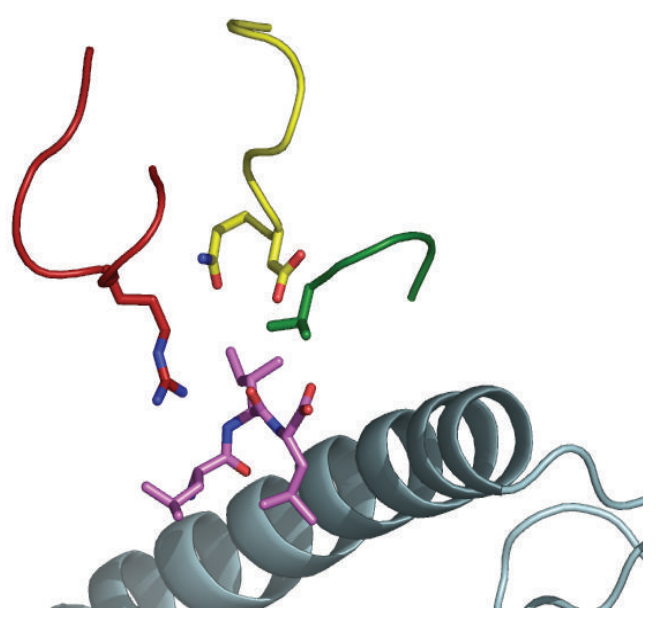

(b)

FIGURE 2: Crystal structure of the HLA-E/KK50.4 TCR complex [12]. (a) Overview of the HLA-E/KK50.4 TCR structure showing two orthogonal views. The HLA-E heavy chain is shown in grey ribbons, the UL40 peptide in pink sticks, and $\beta 2 \mathrm{~m}$ in blue ribbons. The KK50.4 alpha and beta chains are shown as orange and green ribbons, respectively. (b) The convergence of all 3 CDR $\beta$ loops onto P8 Ile of the peptide bound to HLA-E. The HLA-E heavy chain is grey, peptide is pink, CDR1 $\beta$ is yellow, CDR $2 \beta$ is green, and CDR $3 \beta$ is shown in red.

class Ia alleles $[34,35,42]$ (Table 1 ). It is a priori not clear why healthy human subjects would mount T-cell responses towards signal sequences of conserved class Ia molecules. While some of these studies lack information on the HLAtyping of the donors, others have suggested that these cells are mostly reactivity against nonself target peptides of signal sequences [34, 35, 42]. Similarly, cytotoxic $\mathrm{CD}^{+}$T-cells recognizing HLA-E binding sequences from TCR V $\beta$ chains were detected in peripheral blood [33]. An alternative, nonexclusive, explanation may be that these T-cells are reactive with nonself virally derived antigens that share sequence homology with the MHC class Ia derived signal sequences used in these studies.

Involvement of the TCR in specific recognition of HLAE was derived from "NK-CTL" clones. Although these Tcells were originally termed "NK-CTL" due to their ability to lyse a broad range of allogeneic targets, it was subsequently found that these T-cells were specific for the CMV UL40encoded peptide (VMAPRTLIL) bound to HLA-E [35, 42]. The detected alloreactivity was due to target cells possessing HLA-C alleles encoding the same sequence as the UL40 peptide (e.g., HLA-C ${ }^{*} 03$ ). UL40-specific T-cells can reach frequencies in the circulation similar to those restricted by classical HLA-I, indicating their potential to play a significant role in CMV immunity [50].

The TCR from an UL40-specific T-cell clone, KK50.4, was cloned, expressed, and analysed for its interaction with HLA-E in complex with the UL40-epitope VMAPRTLIL (Figure 2) [12]. Overall, the structural basis for recognition of HLA-E largely overlaps that of TCR recognition of HLA class Ia. However, in order for UL40-specific T-cells to recognize the UL40 antigen or allogeneic HLA-I peptides, the UL40-specific TCR need to distinguish between the UL40 epitope (VMAPRTLIL) and nearly identical self peptides which may differ by as little as a single methyl group (e.g., VMAPRTLVL). Analysis of TCR sequences from UL40 specific T-cell clones suggested there were a limited number of TCRs capable of such discrimination, as all of the clones isolated utilized TRBV14 $(\mathrm{V} \beta 16)$ and there was a characteristic arginine residue present in the CDR3 $\beta$ (Figure 2) [12]. Structural analyses of the KK50.4 clone showed that the convergence of CDR1, CDR2, and CDR3 of the KK50.4 $\beta$ chain onto P8 Ile determined self/nonself discrimination (Figure 2). Notably, the highly selected arginine present in the CDR3 $\beta$ made multiple contacts with both HLA-E and peptide.

As the CMV UL40 peptide is highly homologous to HLA class Ia derived signal sequences, typically these T-cells are only observed in individuals where the UL40 epitope differs from that found in self-HLA-C alleles (e.g., HLA$C^{*} 07$ homozygotes) [12]. However, other pathogen-derived peptides that bind to HLA-E are more different from the class Ia signal sequences and may therefore depend less on the donors' HLA class Ia genotype.

(2) HLA-E and HIV. HIV-nef proteins interact with the intracellular domain of HLA-A and HLA-B molecules, resulting in downregulation of HLA class Ia molecules from the cell surface. In contrast, HLA-C and HLA class Ib molecules, particularly, HLA-E and HLA-G, lack these intracellular nefinteraction domains and thus remain expressed normally on the cell surface of HIV infected cells [51]. In addition, it has been shown that a peptide from the HIV-1 capsid protein p24 (AISPRTLNA) may further enhance HLA-E surface expression [52]. However, recently it was shown that this peptide presented in HLA-E is not recognized by CD94/NKG2A and that these cells thus were not protected against NK mediated T-cell lysis [53]. This is most likely due to lack of homology between the HIV p24 peptide and HLA leader peptides, which prevents ligation of CD94NKG2A to the HLA-E peptide complex [53]. Inhibition of NK mediated lysis of HIV-1 infected T-cells rather appears to be 
the result of HLA-C expression and recognition by NK cells that specifically express the NK cell receptors KIR2DL1/2/3 [53]. Interestingly, it has not yet been investigated whether the HIV-1 p24 peptide, presented in HLA-E, may be recognized by the host adaptive immune system and thus may result in specific $\mathrm{CD}^{+}{ }^{+}$-cells. Induction of such $\mathrm{CD}^{+} \mathrm{T}$-cell responses would be interesting from a therapeutic as well as vaccination point of view.

In rhesus macaques, MHC-E (or Mamu-E) is the homologue of human HLA-E, which also showed upregulated expression in HIV/SIV infected animals [28]. Vaccination of rhesus macaques with a CMV-based vector, expressing HIV gag, revealed a very strong $\mathrm{CD} 8^{+} \mathrm{T}$-cell response $(\alpha \beta$ TCR) with a large variety of specific interactions, with an estimated induction of 4 distinct epitopes per 100 amino acids in all tested HIV/SIV derived antigens [28]. Although this massive MHC-E restricted response was due to the specific design of the viral vector, it clearly illustrates the abundance of potential HLA-E epitopes in a large array of antigens. Detailed characterization of the peptide-binding domain revealed a relatively open structure, exposing many side chains for interaction with the TCR [28]. Interestingly, next to inducing MHC-E restricted T-cells, these CMV recombinant vectors also induced a significant population of other unconventional $\mathrm{CD}^{+} \mathrm{T}$-cells, which were restricted by MHC class II molecules.

2.2.2. Bacterial Antigens. Bacteria like Salmonella and Mtb are intracellular pathogens that hijack host cells to promote their own survival. Intriguingly, the expression of HLA-E is enriched on Mtb phagosomes compared to classical HLA class Ia family members, thus presumably facilitating HLAE loading by Mtb peptides in infected cells [29]. In 1998, Lewinsohn et al. identified Mtb specific $\mathrm{CD}^{+} \mathrm{T}$-cell clones that appeared to be restricted to MHC class Ib [31], two of which were HLA-E restricted [32]. However the Mtb derived peptide ligands were not identified. Unpublished recent data point to a peptide derived from the Mtb glycoprotein Mpt32 (David Lewinsohn, personal communication, manuscript in preparation). In an independent effort, we have screened the Mtb genome for the presence of peptides that could potentially be presented by HLA-E and selected 69 peptides based on 3 different prediction algorithms [30]. Many of these peptides were recognized by donors that had been previously sensitized by mycobacteria, suggesting in vivo priming and T-cell memory for several HLA-E epitopes [30]. We have shown that these peptides presented in HLA-E elicit $\mathrm{CD}^{+}{ }^{+}$T-cell activation through the TCR, as measured by both ZAP70 phosphorylation (the first downstream effect in TCR signalling), as well as CD137 expression (a molecule exclusively expressed following specific antigen recognition on $\mathrm{CD}^{+}$T-cells) [47]. Moreover, HLA-E restricted Tcell lines specific for Mtb had strongly reduced cytokine production in the presence of blocking antibodies against the $\alpha \beta$ TCR or HLA-E [48]. Altogether, these data support specific recognition of HLA-E peptide complexes by Mtb specific TCRs. HLA-E binding peptides from Mtb were not capable of preventing NK mediated lysis in a CD94/NKG2A dependent manner [48], similar to HIV p24 [53], indicating that only surface expression of peptide containing HLA-E may not be sufficient.

Detailed characterization of these T-cells revealed that they are cytolytic or suppressive, and that T-cells reactive against the same peptide can display different functional polarities, indicating that polarity is not determined by the peptide. Interestingly, T-cell clones with cytolytic activity were also capable of inhibiting intracellular outgrowth of Mtb, suggesting that they are potent antimycobacterial effector cells [47]. Many of the HLA-E restricted Mtb specific Tcells did not produce typical cytotoxic T-lymphocyte associated cytokines, nor did they produce classical Thl cytokines (IFN $\gamma$, TNF, and IL2), but instead they produced an array of Th2 cytokines including IL-4, IL-5, IL-10, and IL-13, as well as the Th2 associated transcription factor GATA-3 (Table 2) [47, 48]. In patients with TB disease, HLA-E tetramers identified Mtb specific HLA-E restricted CD8 ${ }^{+}$T-cells, with the highest frequencies at $\mathrm{TB}$ diagnosis and waning of the response during successful treatment [48]. Moreover, in line with the knowledge that HLA-E is not susceptible to downregulation by HIV, we were able to detect HLA-E specific T-cells in patients that concomitantly were infected with Mtb and HIV [48].

Salmonella peptides presented by HLA-E are also recognized by HLA-E restricted T-cells. Volunteers vaccinated with a $S$. typhi vaccine had a robust HLA-E restricted T-cell response, as measured by the cytolytic capacities of these cells, such as granzyme B activity (Table 1) [43]. Kinetic analysis of these responses in a similarly vaccinated cohort revealed that the HLA-E restricted T-cells are long-lasting, up to 2 years after vaccination, again suggesting immune memory [46]. Moreover, following challenge experiments with Salmonella in unvaccinated, healthy volunteers, multifunctional HLAE restricted $\mathrm{CD}^{+}$T-cells were detected and correlated with protection against typhoid disease development [49].

2.2.3. Tumor and Self-Antigens. Interestingly, T-cells reactive with self Hsp60sp presented by HLA-E have also been identified, both in healthy donors and in patients with type 1 diabetes [45]. These $\mathrm{CD}^{+} \mathrm{T}$-cell lines were involved in discriminating self from nonself in the periphery, and defective discrimination between self and nonself was detected in the majority of patients with type 1 diabetes [45]. While many viruses are known to interfere with antigen processing and presentation, resulting in peptide presentation in a TAPindependent manner, this is also the case in many tumors. As a consequence, tumor unique antigens may be presented also in the context of HLA-E $[54,55]$, which may subsequently be recognized by cytotoxic T-cells [55]. In contrast to what was expected, in humans the presence of CTLs was only beneficial in patients with lung carcinoma if HLA-E was not expressed by the tumor, indicating that HLA-E restricted CTLs may not directly contribute to tumor elimination in these patients [56].

2.2.4. Autoimmune Diseases. HLA-E restricted T-cell responses have been studied only to a very limited extent in autoimmune diseases; however they could potentially play an important role. In patients with multiple sclerosis (MS), 


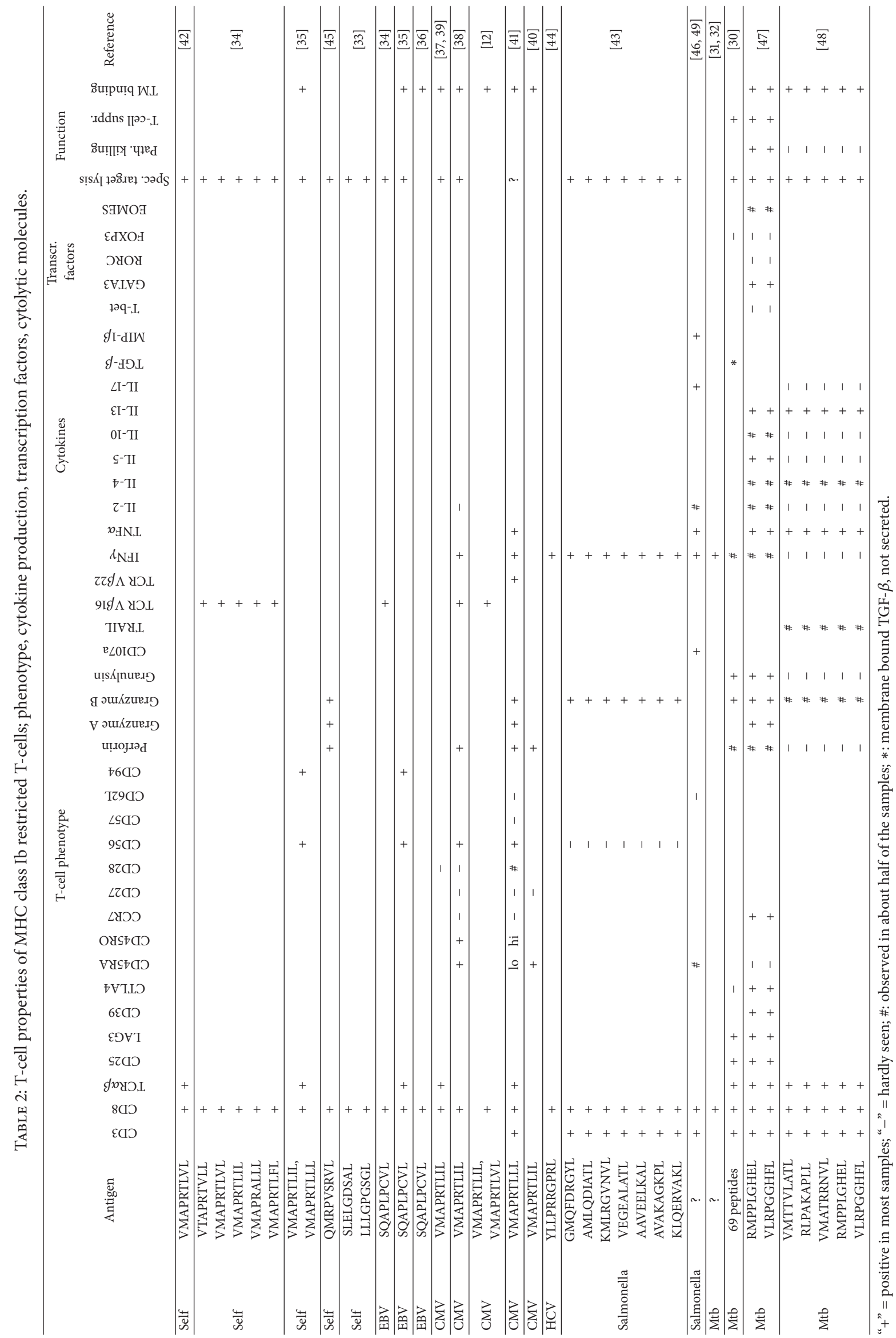


increased frequencies of EBV specific, HLA-E restricted $\mathrm{CD}^{+} \mathrm{T}$-cells have been found to be associated mostly with the relapsing remitting form of the disease rather than the progressive form [36]. Moreover, $\mathrm{CD} 8^{+} \mathrm{T}$-cells induced by glatiramer acetate vaccination appear to be restricted to HLA-E and have immunomodulatory capacities, resulting in amelioration of MS [57-59]. HLA-E restricted CD8 ${ }^{+}$T-cells in patients with MS appeared phenotypically different from healthy controls; however these T-cells were selected based on the expression of NKG2C and thus may reflect only a small subset of HLA-E restricted T-cells [60]. In rheumatoid arthritis (RA), limited information is available, although HLA-E polymorphisms may be associated with disease susceptibility and treatment responsiveness [61]. Interestingly and similar to the studies in MS, RA like autoimmunity can be strongly inhibited by induction of (self Hsp60) peptide specific Qa-1 restricted suppressor T-cells in mice [62]. Furthermore, there is a defect in $\mathrm{CD}^{+}{ }^{+} \mathrm{T}$-cell recognition of HLA-E/Hsp60sp in patients with type I diabetes [45]. Thus, MHC-E restricted $\mathrm{CD}^{+}$T-cells with immunoregulatory properties may be critical in amelioration of autoimmune diseases and deserve further detailed characterization.

2.3. Properties of HLA-E Restricted Human T-Cells. Surprisingly, little information is available on the phenotype and function of human $\mathrm{CD} 8^{+} \mathrm{T}$-cells recognizing peptides presented by HLA-E. In many studies, HLA-E restricted Tcells have only been enumerated using tetramer staining, or the presence of HLA-E reactivity was demonstrated using cytolytic assays (Table 2). Analyses of the TCR composition were only performed in a limited number of studies and, as mentioned above, found consistent selection of TRAV14 (V $\beta 16)$ in CMV specific TCRs [12]. Additional studies have also determined V $\beta 22$ usage in CMV restricted TCRs [41].

Basic descriptive information of HLA-E restricted Tcells, such as memory phenotype, is also largely lacking. The limited data that have been published do not suggest specific memory stages to be overrepresented among HLAE restricted $\mathrm{CD}^{+} \mathrm{T}$-cell populations, since both CD45RA positive and negative populations were identified, as was also reported for CCR7 (Table 2). Generally, the T-cells reported expressed cytolytic molecules, but in some cases these were weakly expressed or even undetectable (Table 2). Specific target cell lysis was frequently used as read out to demonstrate HLA-E restriction of $\mathrm{CD} 8^{+}$T-cells. Recently, we demonstrated for the first time that HLA-E restricted $\mathrm{CD}^{+}$T-cell clones had antibacterial activity against Mtb, considered to be an important property in immune control of intracellular pathogens (Table 2). Other studies have not assessed or reported viral or bacterial inhibition following HLA-E restricted $\mathrm{CD}^{+}$T-cell activation or downstream target cell lysis. It will be of interest to identify the mechanism of control of intracellular outgrowth of Mtb, as this could be a yet unknown component of the immune system that could be harnessed for preventive or therapeutic interventions.

Cytokine production has been analysed in detail only in the most recent series of papers. Originally, studies focused on the production of classical Thl cytokines, such as IFN $\gamma$ and $\mathrm{TNF} \alpha$, and although sometimes detected in HLA-E restricted T-cells, not all produced IFN $\gamma$ in response to specific peptide stimulation (Table 2). As mentioned above, we recently found Mtb specific HLA-E restricted $\mathrm{CD} 8^{+} \mathrm{T}$-cells to produce $T h 2$ rather than Th1 cytokines and demonstrated that these cells utilize IL-4 to activate B-cells (Table 2). It would be interesting and relevant to investigate $\mathrm{Th} 2$ cytokine production also in HLA-E restricted $\mathrm{CD} 8^{+} \mathrm{T}$-cells in response to other ligands. Moreover, an analysis of transcription factor expression in HLA-E restricted CD ${ }^{+} \mathrm{T}$-cells has been limited thus far to our description of Mtb specific T-cells expressing GATA-3 (Table 2).

One of the major differences between innate and adaptive immunity is the formation of immunological memory during adaptive immune responses. As HLA-E restricted $\mathrm{CD} 8^{+} \mathrm{T}-$ cells are activated through their TCR, in an antigen specific manner, it is likely that they also differentiate into memory cells. The formation of memory cells following HLA-E mediated antigen presentation would be an important prerequisite for successful application of these peptides in future vaccination strategies. However, information on T-cell memory development is limited. Phenotypically, effector memory [30] and effector memory recently activated [38, 41] have been described as indicators of memory. Moreover, screening for recognition of HLA-E restricted Mtb peptides by human donors showed recognition only in donors that had been sensitized by mycobacteria (as measured by PPD recognition), suggesting that in vivo priming, and thus memory induction, was critical [30]. Vaccination of healthy volunteers with a single dose of $S$. typhi strain Ty2la resulted in antigen specific, HLA-E restricted $\mathrm{CD}^{+}{ }^{+} \mathrm{T}$-cells that were detectable up to 2 years after vaccination, suggesting that HLA-E restricted memory T-cells had been induced [46]. Also, individuals with latent TB and individuals successfully treated for TB disease still had circulating $\mathrm{CD} 8^{+} \mathrm{T}$-cells binding to HLA-E tetramers (TM) loaded with Mtb peptides (notwithstanding that the frequencies detected were highest in patients with active TB disease); the persistence of responses after microbiological cure thus also suggests immune memory [48].

2.4. Qa- $1^{b}$ : The Murine HLA-E Homologue. The murine homologue of human HLA-E is Qa- $1^{\mathrm{b}}$, which also presents signal sequences from $\mathrm{MHC}$ class I proteins that are called Qdm (Qa-1 determinant modifier) [54]. Qa- ${ }^{\mathrm{b}}$ can also bind CD94/NKG2A complexes to silence NK cell activity. However, also specific recognition of Qa- $1^{\mathrm{b}}$ peptide complexes by T-cells has been described [54]. Interestingly, similar to HLA$\mathrm{E}, \mathrm{Qa}-\mathrm{1}^{\mathrm{b}}$ restricted T-cells have been isolated that are able to detect differences in leader-sequenced derived peptides between mouse strains [63], although there is no indication they are involved in immunity to mouse CMV. Also, similar to HLA-E, Qa- $1^{\mathrm{b}}$ restricted T-cells have been implicated in immunity to pathogens, including Listeria monocytogenes [64] and Salmonella typhimurium [65].

Qa- ${ }^{\mathrm{b}}$ restricted $S$. typhimurium specific T-cells are curiously cross-reactive to self Hsp-60 derived peptides and thus have been implicated in autoimmune conditions [66]. Furthermore, there is a large and older body of research focusing on $\mathrm{Qa}-1^{\mathrm{b}}$ restricted suppressor $\mathrm{CD} 8^{+} \mathrm{T}$-cells. These T-cells 
reportedly recognize Qa-1 ${ }^{\mathrm{b}}$ in a TCR dependent manner and act to suppress autoreactive $\mathrm{CD} 4^{+} \mathrm{T}$-cells, thereby attenuating the development of autoimmune encephalomyelitis (a mouse model of multiple sclerosis), very similar to the role of HLA-E restricted $\mathrm{CD}^{+}$T-cells evoked by vaccination in human MS [67-69]. In this model, Qa- ${ }^{\mathrm{b}}$ deficient mice developed exaggerated secondary $\mathrm{CD} 4$ responses, as a result of the lack of a population of regulatory $\mathrm{CD}^{+} \mathrm{T}$-cells, demonstrating the in vivo significance of suppressor Qa- $1^{\mathrm{b}}$ restricted T-cells. Qa- $1^{\mathrm{b}}$ restricted T-cells appear critical for the maintenance of self-tolerance. Their role in infectious diseases and in particular in the elimination of pathogens has not been studied in great detail thus far. Moreover, a detailed description of the phenotype and function of these cells is lacking and warrants further investigation.

\section{Summary and Implications}

HLA-E plays a dual role in the innate and adaptive immune system (Figure 1). The low polymorphism in HLA-E in conjunction with its relative insensitivity to downregulation by, for example, HIV, makes HLA-E an interesting target for vaccination strategies against infectious diseases and tumors. Small sets of peptides should suffice to induce T-cells recognizing foreign antigens and mount effector responses. The quality of these T-cell responses needs further investigation, however, given the diversity in functions that have been described thus far, ranging from cytotoxicity and pathogen control to immune suppression. Various pathogen-derived antigens can bind and stabilize HLA-E at the cell surface, for some of which this may be an essential mechanism to prevent NK mediated target cell lysis. However, many of these antigens can also be recognized in a TCR dependent manner by $\mathrm{CD} 8^{+} \mathrm{T}$-cells. These T-cells may suppress bystander Tcells or lyse (infected) target cells and inhibit intracellular bacteria, indicating an important functional contribution to the immune response. Nevertheless, the relative frequency of HLA-E restricted T-cells and their in vivo relevance in many cases remains unknown and unstudied. Although these T-cells are donor-unrestricted, they in many aspects display similar functionalities to classical, conventional T-cells.

Pathogen specific HLA-E restricted $\mathrm{CD} 8^{+} \mathrm{T}$-cells are an interesting new player in the field of immunology. Future work should address their exact roles and relative contributions in the immune response against infectious diseases.

\section{Competing Interests}

The authors declare that they have no competing interests.

\section{References}

[1] H. D. Moreau and P. Bousso, "Visualizing how T cells collect activation signals in vivo," Current Opinion in Immunology, vol. 26, no. 1, pp. 56-62, 2014.

[2] F. Benvenuti, "The dendritic cell synapse: a life dedicated to $\mathrm{t}$ cell activation," Frontiers in Immunology, vol. 7, article 70, 2016.
[3] J. G. P. Sissons and M. B. A. Oldstone, "Killing of virus-infected cells by cytotoxic lymphocytes," Journal of Infectious Diseases, vol. 142, no. 1, pp. 114-119, 1980.

[4] P. Escoll, M. Rolando, and C. Buchrieser, "Modulation of host autophagy during bacterial infection: sabotaging host munitions for pathogen nutrition," Frontiers in Immunology, vol. 7, article 81, 2016.

[5] D. I. Godfrey, A. P. Uldrich, J. Mccluskey, J. Rossjohn, and D. B. Moody, "The burgeoning family of unconventional T cells," Nature Immunology, vol. 16, no. 11, pp. 1114-1123, 2015.

[6] Anthony Nolan Research Institute, HLA Class I and Class II Sequence Alignments, June 2009 Updates, 2009, http://www.ebi .ac.uk/ipd/imgt/hla/.

[7] M. Ulbrecht, T. Honka, S. Person, J. P. Johnson, and E. H. Weiss, "The HLA-E gene encodes two differentially regulated transcripts and a cell surface protein," Journal of Immunology, vol. 149, no. 9, pp. 2945-2953, 1992.

[8] S. Coupel, A. Moreau, M. Hamidou, V. Horejsi, J.-P. Soulillou, and B. Charreau, "Expression and release of soluble HLA-E is an immunoregulatory feature of endothelial cell activation," Blood, vol. 109, no. 7, pp. 2806-2814, 2007.

[9] P. Tomasec, V. M. Braud, C. Rickards et al., "Surface expression of HLA-E, an inhibitor of natural killer cells, enhanced by human cytomegalovirus gpUL40," Science, vol. 287, no. 5455, pp. 1031-1033, 2000.

[10] I. Foroni, A. R. Couto, B. F. Bettencourt, M. Santos, M. Lima, and J. Bruges-Armas, "HLA-E, HLA-F and HLA-G-the nonclassical side of the MHC cluster," in HLA and Associated Important Diseases, Y. Xi, Ed., chapter 3, pp. 61-109, InTech, Rijeka, Croatia, 2014.

[11] R. Apps, S. P. Murphy, R. Fernando, L. Gardner, T. Ahad, and A. Moffett, "Human leucocyte antigen (HLA) expression of primary trophoblast cells and placental cell lines, determined using single antigen beads to characterize allotype specificities of anti-HLA antibodies," Immunology, vol. 127, no. 1, pp. 26-39, 2009.

[12] H. L. Hoare, L. C. Sullivan, G. Pietra et al., "Structural basis for a major histocompatibility complex class Ib-restricted $\mathrm{T}$ cell response," Nature Immunology, vol. 7, no. 3, pp. 256-264, 2006.

[13] V. M. Braud, D. S. J. Allan, C. A. O'Callaghan et al., "HLA-E binds to natural killer cell receptors CD94/NKG2A, B and C," Nature, vol. 391, no. 6669, pp. 795-799, 1998.

[14] C. Grimsley and C. Ober, "Population genetic studies of HLA-E: evidence for selection," Human Immunology, vol. 52, no. 1, pp. 33-40, 1997.

[15] T. Kraemer, R. Blasczyk, and C. Bade-Doeding, "HLA-E: a novel player for histocompatibility," Journal of Immunology Research, vol. 2014, Article ID 352160, 7 pages, 2014.

[16] R. K. Strong, M. A. Holmes, P. Li, L. Braun, N. Lee, and D. E. Geraghty, "HLA-E allelic variants: correlating differential expression, peptide affinities, crystal structures, and thermal stabilities," Journal of Biological Chemistry, vol. 278, no. 7, pp. 5082-5090, 2003.

[17] T. Kraemer, A. A. Celik, T. Huyton, H. Kunze-Schumacher, R. Blasczyk, and C. Bade-Döding, "HLA-E: presentation of a broader peptide repertoire impacts the cellular immune response-implications on HSCT outcome," Stem Cells International, vol. 2015, Article ID 346714, 12 pages, 2015.

[18] A. A. Celik, T. Kraemer, T. Huyton, R. Blasczyk, and C. Bade-Döding, "The diversity of the HLA-E-restricted peptide repertoire explains the immunological impact of the Arg107Gly mismatch," Immunogenetics, vol. 68, no. 1, pp. 29-41, 2016. 
[19] C. A. O’Callaghan, J. Tormo, B. E. Willcox et al., "Structural features impose tight peptide binding specificity in the nonclassical MHC molecule HLA-E," Molecular Cell, vol. 1, no. 4, pp. 531-541, 1998.

[20] H. L. Hoare, L. C. Sullivan, C. S. Clements et al., "Subtle changes in peptide conformation profoundly affect recognition of the non-classical MHC class I molecule HLA-E by the CD94-NKG2 natural killer cell receptors," Journal of Molecular Biology, vol. 377, no. 5, pp. 1297-1303, 2008.

[21] M. Ulbrecht, S. Modrow, R. Srivastava, P. A. Peterson, and E. H. Weiss, "Interaction of HLA-E with peptides and the peptide transporter in vitro: implications for its function in antigen presentation," Journal of Immunology, vol. 160, no. 9, pp. 43754385, 1998.

[22] M. Ulbrecht, S. Martinozzi, M. Grzeschik et al., "Cutting edge: the human cytomegalovirus UL40 gene product contains a ligand for HLA-E and prevents NK cell-mediated lysis," The Journal of Immunology, vol. 164, no. 10, pp. 5019-5022, 2000.

[23] J. Nattermann, H. D. Nischalke, V. Hofmeister et al., "The HLAA2 restricted T cell epitope HCV core $_{35-44}$ stabilizes HLA-E expression and inhibits cytolysis mediated by natural killer cells," The American Journal of Pathology, vol. 166, no. 2, pp. 443-453, 2005.

[24] J. Stevens, E. Joly, J. Trowsdale, and G. W. Butcher, "Peptide binding characteristics of the non-classical class Ib MHC molecule HLA-E assessed by a recombinant random peptide approach," BMC Immunology, vol. 2, no. 1, article 5, 2001.

[25] J. D. Miller, D. A. Weber, C. Ibegbu, J. Pohl, J. D. Altman, and P. E. Jensen, "Analysis of HLA-E peptide-binding specificity and contact residues in bound peptide required for recognition by CD94/NKG2," Journal of Immunology, vol. 171, no. 3, pp. 13691375, 2003.

[26] V. Braud, E. Y. Jones, and A. McMichael, "The human major histocompatibility complex class Ib molecule HLA-E binds signal sequence-derived peptides with primary anchor residues at positions 2 and 9," European Journal of Immunology, vol. 27, no. 5, pp. 1164-1169, 1997.

[27] M. H. Lampen, C. Hassan, M. Sluijter et al., "Alternative peptide repertoire of HLA-E reveals a binding motif that is strikingly similar to HLA-A2," Molecular Immunology, vol. 53, no. 1-2, pp. 126-131, 2013.

[28] S. G. Hansen, H. L. Wu, B. J. Burwitz et al., "Broadly targeted $\mathrm{CD}^{+} \mathrm{T}$ cell responses restricted by major histocompatibility complex E," Science, vol. 351, no. 6274, pp. 714-720, 2016.

[29] J. E. Grotzke, M. J. Harriff, A. C. Siler et al., “The Mycobacterium tuberculosis phagosome is a HLA-I processing competent organelle," PLoS Pathogens, vol. 5, no. 4, Article ID e1000374, 2009.

[30] S. A. Joosten, K. E. van Meijgaarden, P. C. van Weeren et al., "Mycobacterium tuberculosis peptides presented by HLA-E molecules are targets for human $\mathrm{CD}^{+} \mathrm{T}$-cells with cytotoxic as well as regulatory activity," PLoS Pathogens, vol. 6, no. 2, Article ID e1000782, 2010.

[31] D. M. Lewinsohn, M. R. Alderson, A. L. Briden, S. R. Riddell, S. G. Reed, and K. H. Grabstein, "Characterization of human $\mathrm{CD}^{+} \mathrm{T}$ cells reactive with Mycobacterium tuberculosisinfected antigen-presenting cells," Journal of Experimental Medicine, vol. 187, no. 10, pp. 1633-1640, 1998.

[32] A. S. Heinzel, J. E. Grotzke, R. A. Lines et al., "HLA-E-dependent presentation of Mtb-derived antigen to human $\mathrm{CD}^{+} \mathrm{T}$ cells," The Journal of Experimental Medicine, vol. 196, no. 11, pp. 14731481, 2002.
[33] J. Li, I. Goldstein, E. Glickman-Nir, H. Jiang, and L. Chess, "Induction of TCR V $\beta$-specific CD $8+$ CTLs by TCR V $\beta$-derived peptides bound to HLA-E," Journal of Immunology, vol. 167, no. 7, pp. 3800-3808, 2001.

[34] C. Romagnani, G. Pietra, M. Falco et al., "Identification of HLA-E-specific alloreactive T lymphocytes: a cell subset that undergoes preferential expansion in mixed lymphocyte culture and displays a broad cytolytic activity against allogeneic cells," Proceedings of the National Academy of Sciences of the United States of America, vol. 99, no. 17, pp. 11328-11333, 2002.

[35] P. García, M. Llano, A. B. de Heredia et al., "Human T cell receptor-mediated recognition of HLA-E," European Journal of Immunology, vol. 32, no. 4, pp. 936-944, 2002.

[36] P. B. Jørgensen, A. H. Livbjerg, H. J. Hansen, T. Petersen, and P. Höllsberg, "Epstein-Barr virus peptide presented by HLA$\mathrm{E}$ is predominantly recognized by $\mathrm{CD} 8^{\text {bright }}$ cells in multiple sclerosis patients," PLoS ONE, vol. 7, no. 9, Article ID e46120, 2012.

[37] G. Pietra, C. Romagnani, P. Mazzarino et al., "HLA-E-restricted recognition of cytomegalovirus-derived peptides by human $\mathrm{CD}^{+}$cytolytic T lymphocytes," Proceedings of the National Academy of Sciences of the United States of America, vol. 100, no. 19, pp. 10896-10901, 2003.

[38] P. Mazzarino, G. Pietra, P. Vacca et al., "Identification of effectormemory CMV-specific T lymphocytes that kill CMV-infected target cells in an HLA-E-restricted fashion," European Journal of Immunology, vol. 35, no. 11, pp. 3240-3247, 2005.

[39] C. Romagnani, G. Pietra, M. Falco, P. Mazzarino, L. Moretta, and M. C. Mingari, "HLA-E-restricted recognition of human cytomegalovirus by a subset of cytolytic T lymphocytes," Human Immunology, vol. 65, no. 5, pp. 437-445, 2004.

[40] L. C. Sullivan, G. P. Westall, J. M. L. Widjaja et al., "The presence of HLA-E-restricted, CMV-specific $\mathrm{CD}^{+} \mathrm{T}$ cells in the blood of lung transplant recipients correlates with chronic allograft rejection," PLoS ONE, vol. 10, no. 8, Article ID e0135972, 2015.

[41] M. Allard, P. Tonnerre, S. Nedellec et al., "HLA-E-restricted cross-recognition of allogeneic endothelial cells by CMVassociated CD8 T cells: a potential risk factor following transplantation," PLoS ONE, vol. 7, no. 11, Article ID e50951, 2012.

[42] G. Pietra, C. Romagnani, M. Falco et al., "The analysis of the natural killer-like activity of human cytolytic T lymphocytes revealed HLA-E as a novel target for TCR $\alpha / \beta$-mediated recognition," European Journal of Immunology, vol. 31, no. 12, pp. 3687-3693, 2001.

[43] R. Salerno-Gonçalves, M. Fernandez-Viña, D. M. Lewinsohn, and M. B. Sztein, "Identification of a human HLA-E-restricted $\mathrm{CD}^{+} \mathrm{T}$ cell subset in volunteers immunized with Salmonella enterica serovar Typhi strain Ty21a typhoid vaccine," Journal of Immunology, vol. 173, no. 9, pp. 5852-5862, 2004.

[44] D. Schulte, M. Vogel, B. Langhans et al., “The HLA-ER/HLA-ER genotype affects the natural course of hepatitis $\mathrm{C}$ virus (HCV) infection and is associated with HLA-E-Restricted recognition of an HCV-Derived peptide by interferon- $\gamma$-secreting human $\mathrm{CD}^{+}$T cells," Journal of Infectious Diseases, vol. 200, no. 9, pp. 1397-1401, 2009.

[45] H. Jiang, S. M. Canfield, M. P. Gallagher et al., "HLA-Erestricted regulatory $\mathrm{CD}^{+} \mathrm{T}$ cells are involved in development and control of human autoimmune type 1 diabetes," The Journal of Clinical Investigation, vol. 120, no. 10, pp. 3641-3650, 2010.

[46] R. Salerno-Goncalves, R. Wahid, and M. B. Sztein, "Ex vivo kinetics of early and long-term multifunctional human leukocyte antigen E-specific $\mathrm{CD} 8^{+}$cells in volunteers immunized 
with the Ty21a typhoid vaccine," Clinical and Vaccine Immunology, vol. 17, no. 9, pp. 1305-1314, 2010.

[47] K. E. van Meijgaarden, M. C. Haks, N. Caccamo, F. Dieli, T. H. M. Ottenhoff, and S. A. Joosten, "Human $\mathrm{CD}^{+}$T-cells recognizing peptides from Mycobacterium tuberculosis (Mtb) presented by HLA-E have an unorthodox Th2-like, multifunctional, $M t b$ inhibitory phenotype and represent a novel human T-cell subset," PLoS Pathogens, vol. 11, no. 3, Article ID e1004671, pp. 1-24, 2015.

[48] N. Caccamo, G. Pietra, L. C. Sullivan et al., "Human CD8 T lymphocytes recognize Mycobacterium tuberculosis antigens presented by HLA-E during active tuberculosis and express type 2 cytokines," European Journal of Immunology, vol. 45, no. 4, pp. 1069-1081, 2015.

[49] S. Fresnay, M. A. McArthur, L. Magder et al., "Salmonella Typhi-specific multifunctional CD8+ T cells play a dominant role in protection from typhoid fever in humans," Journal of Translational Medicine, vol. 14, article 62, 2016.

[50] L. C. Sullivan, C. S. Clements, J. Rossjohn, and A. G. Brooks, "The major histocompatibility complex class Ib molecule HLA$\mathrm{E}$ at the interface between innate and adaptive immunity," Tissue Antigens, vol. 72, no. 5, pp. 415-424, 2008.

[51] G. B. Cohen, R. T. Gandhi, D. M. Davis et al., "The selective downregulation of class I major histocompatibility complex proteins by HIV-1 protects HIV-infected cells from NK cells," Immunity, vol. 10, no. 6, pp. 661-671, 1999.

[52] J. Nattermann, H. D. Nischalke, V. Hofmeister et al., "HIV1 infection leads to increased HLA-E expression resulting in impaired function of natural killer cells," Antiviral Therapy, vol. 10, no. 1, pp. 95-107, 2005.

[53] Z. B. Davis, A. Cogswell, H. Scott et al., "A conserved HIV-1derived peptide presented by HLA-E renders infected T-cells highly susceptible to attack by NKG2A/CD94-bearing natural killer cells," PLoS Pathogens, vol. 12, no. 2, Article ID e1005421, 2016.

[54] T. van Hall, C. C. Oliveira, S. A. Joosten, and T. H. M. Ottenhoff, "The other Janus face of Qa-1 and HLA-E: diverse peptide repertoires in times of stress," Microbes and Infection, vol. 12, no. 12-13, pp. 910-918, 2010.

[55] C. C. Oliveira, P. A. van Veelen, B. Querido et al., “The nonpolymorphic MHC Qa-1b mediates CD8+ T cell surveillance of antigen-processing defects," The Journal of Experimental Medicine, vol. 207, no. 1, pp. 207-221, 2010.

[56] M. Talebian Yazdi, S. van Riet, A. van Schadewijk et al., "The positive prognostic effect of stromal CD8+ tumor-infiltrating $\mathrm{T}$ cells is restrained by the expression of HLA-E in non-small cell lung carcinoma," Oncotarget, vol. 7, no. 3, pp. 3477-3488, 2016.

[57] A. F. Tyler, J. P. Mendoza, M. Firan, and N. J. Karandikar, "CD8 ${ }^{+} \mathrm{T}$ cells are required for glatiramer acetate therapy in autoimmune demyelinating disease," PLoS ONE, vol. 8, no. 6, article e66772, 2013.

[58] S. Sinha, A. W. Boyden, F. R. Itani, M. P. Crawford, and N. J. Karandikar, "CD8 ${ }^{+} \mathrm{T}$-cells as immune regulators of multiple sclerosis," Frontiers in Immunology, vol. 6, article 619, 2015.

[59] D. K. Tennakoon, R. S. Mehta, S. B. Ortega, V. Bhoj, M. K. Racke, and N. J. Karandikar, "Therapeutic induction of regulatory, cytotoxic CD8+ T cells in multiple sclerosis," The Journal of Immunology, vol. 176, no. 11, pp. 7119-7129, 2006.

[60] K. Pannemans, B. Broux, A. Goris et al., "HLA-E restricted $\mathrm{CD}^{+} \mathrm{T}$ cell subsets are phenotypically altered in multiple sclerosis patients," Multiple Sclerosis Journal, vol. 20, no. 7, pp. 790-801, 2014.
[61] M. Iwaszko, J. Świerkot, K. Kolossa, S. Jeka, P. Wiland, and K. Bogunia-Kubik, "Polymorphisms within the human leucocyte antigen-E gene and their associations with susceptibility to rheumatoid arthritis as well as clinical outcome of anti-tumour necrosis factor therapy," Clinical and Experimental Immunology, vol. 182, no. 3, pp. 270-277, 2015.

[62] J. W. Leavenworth, X. Tang, H.-J. Kim, X. Wang, and H. Cantor, "Amelioration of arthritis through mobilization of peptide-specific $\mathrm{CD}^{+}$regulatory T cells," Journal of Clinical Investigation, vol. 123, no. 3, pp. 1382-1389, 2013.

[63] C. J. Aldrich, A. DeCloux, A. S. Woods, R. J. Cotter, M. J. Soloski, and J. Forman, "Identification of a tap-dependent leader peptide recognized by alloreactive T cells specific for a class Ib antigen," Cell, vol. 79, no. 4, pp. 649-658, 1994.

[64] H. G. A. Bouwer, M. S. Seaman, J. Forman, and D. J. Hinrichs, "MHC class Ib-restricted cells contribute to antilisterial immunity: evidence for $\mathrm{Q} a-1 \mathrm{~b}$ as a key restricting element for listeriaspecific CTLs," Journal of Immunology, vol. 159, no. 6, pp. 27952801, 1997.

[65] W.-F. Lo, H. Ong, E. S. Metcalf, and M. J. Soloski, “T cell responses to gram-negative intracellular bacterial pathogens: a role for $\mathrm{CD}^{+} \mathrm{T}$ cells in immunity to Salmonella infection and the involvement of MHC class Ib molecules," Journal of Immunology, vol. 162, no. 9, pp. 5398-5406, 1999.

[66] W.-F. Lo, A. S. Woods, A. DeCloux, R. J. Cotter, E. S. Metcalf, and M. J. Soloski, "Molecular mimicry mediated by MHC class Ib molecules after infection with gram-negative pathogens," Nature Medicine, vol. 6, no. 2, pp. 215-218, 2000.

[67] H. Jiang, S.-L. Zhang, and B. Pernis, "Role of CD8 ${ }^{+} \mathrm{T}$ cells in murine experimental allergic encephalomyelitis," Science, vol. 256, no. 5060, pp. 1213-1215, 1992.

[68] H. Jiang, H. Kashleva, L.-X. Xu et al., "T cell vaccination induces $\mathrm{T}$ cell receptor $\mathrm{V} \beta$-specific Qa-1-restricted regulatory $\mathrm{CD}^{+} \mathrm{T}$ cells," Proceedings of the National Academy of Sciences of the United States of America, vol. 95, no. 8, pp. 4533-4537, 1998.

[69] S. Sarantopoulos, L. Lu, and H. Cantor, "Qa-1 restriction of $\mathrm{CD}^{+}$suppressor T cells," Journal of Clinical Investigation, vol. 114, no. 9, pp. 1218-1221, 2004. 


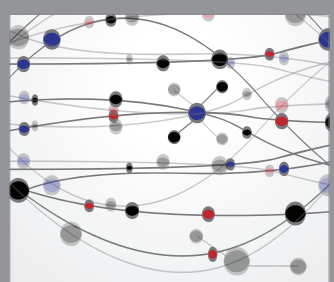

The Scientific World Journal
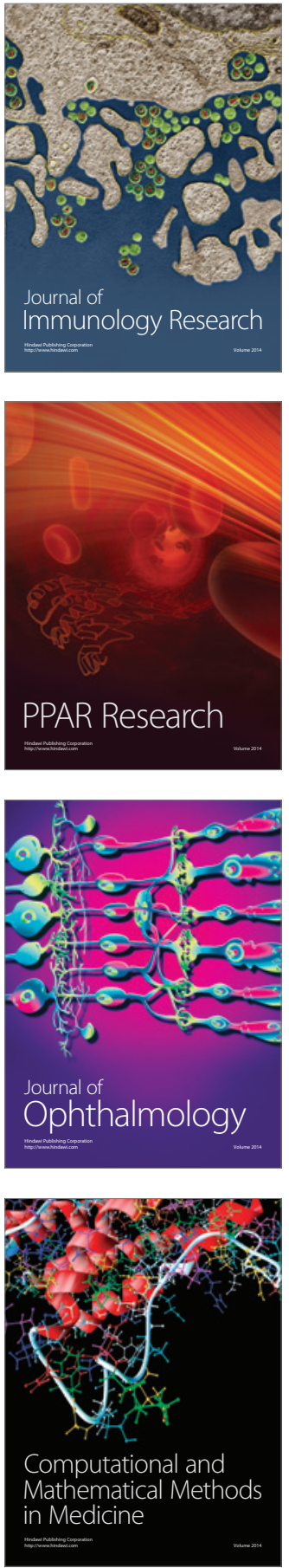

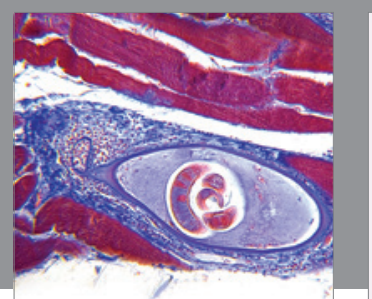

Gastroenterology Research and Practice

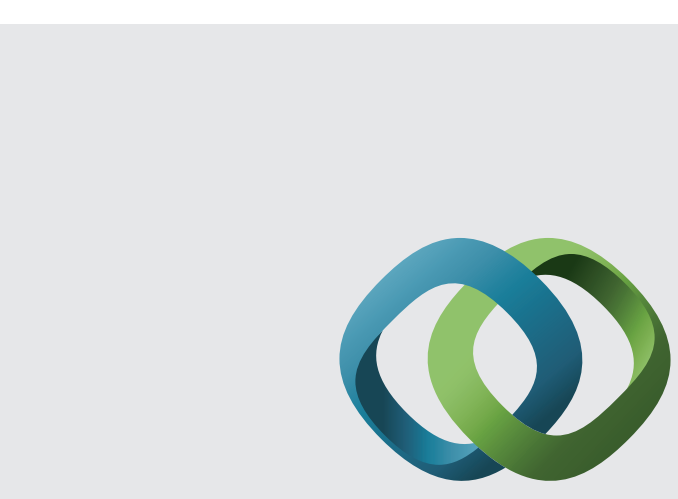

\section{Hindawi}

Submit your manuscripts at

http://www.hindawi.com
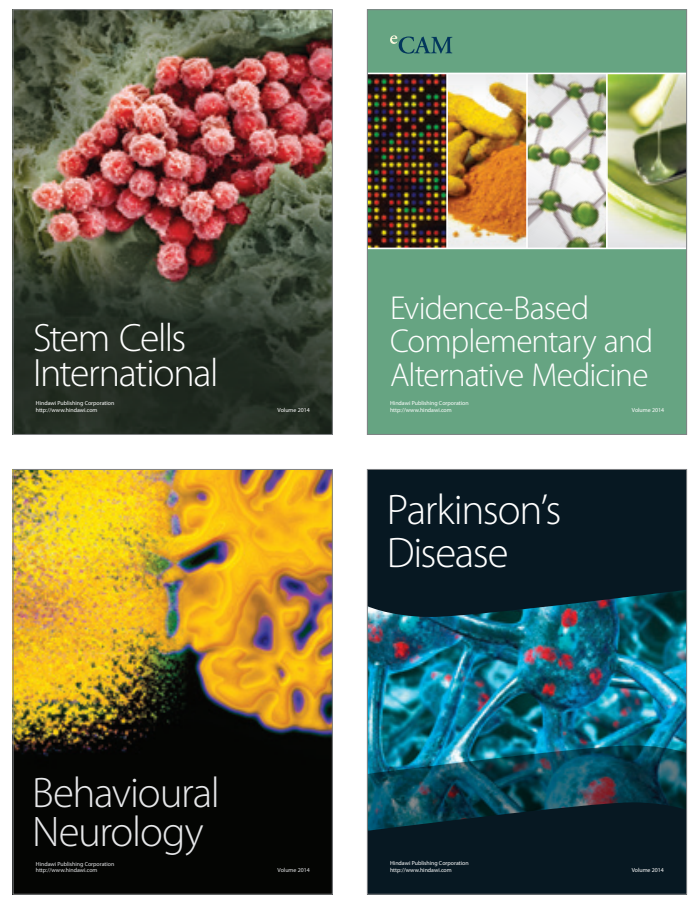
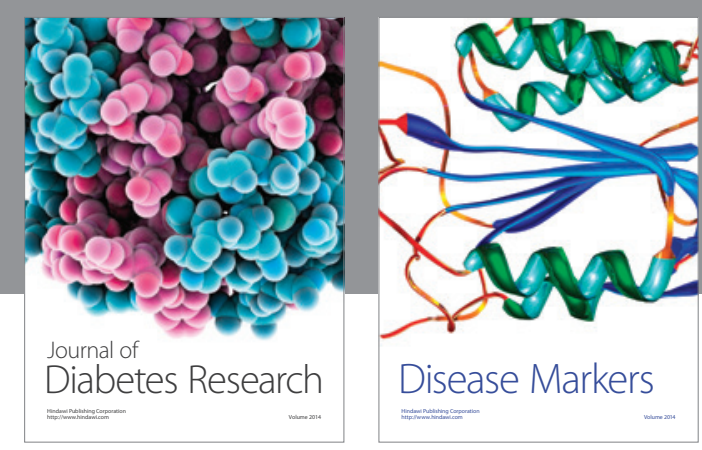

Disease Markers
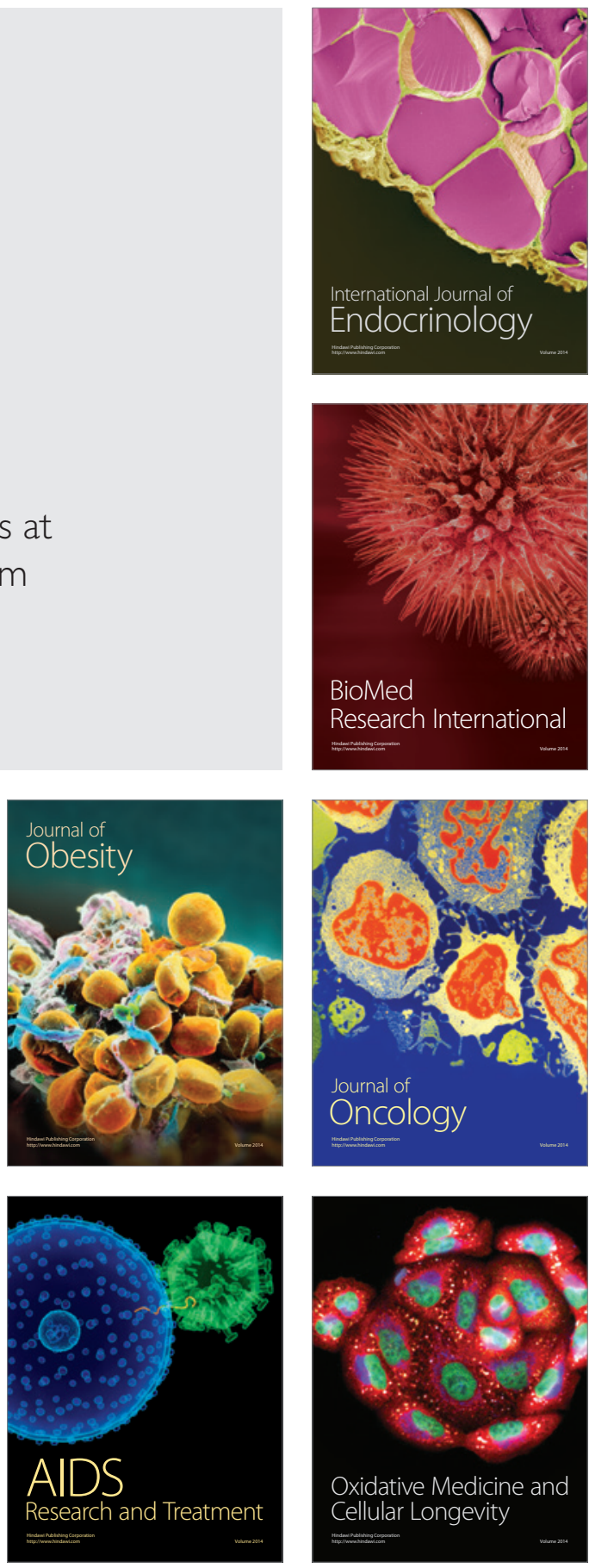